\title{
A viral load-based cellular automata approach to modeling HIV dynamics and drug treatment
}

\author{
Veronica Shi, Abdessamad Tridane, Yang Kuang* \\ Department of Mathematics, Arizona State University, Tempe, AZ 85284, USA
}

Received 15 August 2007; accepted 2 November 2007

Available online 17 November 2007

\begin{abstract}
We formulated a novel cellular automata (CA) model for HIV dynamics and drug treatment. The model is built upon realistic biological processes, including the virus replication cycle and mechanisms of drug therapy. Viral load, its effect on infection rate, and the role of latently infected cells in sustaining HIV infection are among the aspects that are explored and incorporated in the model. We assume that the calculation of the number of cells in the neighborhood which influences the center cell's state is based on the viral load. This variable-cell neighborhood enables the simulation of an infection rate that is correlated to the viral load. This approach leads to a new and flexible way of modeling HIV dynamics and allows for the simulation of different antiretroviral drug treatments based on their individual and combined effects. The results of the simulation show the three phases of HIV dynamics (acute, chronic, and AIDS) and the additional drug response phase when drug treatment is added. The dynamics from the model qualitatively match clinical data. Drug treatment combinations with reverse transcriptase inhibitors and protease inhibitors are simulated using various drug efficacies. The results indicate that the model can be very useful in evaluating different drug therapy regimens.
\end{abstract}

Published by Elsevier Ltd.

\section{Introduction}

In recent years, the combination of mathematical modeling with clinical research has greatly facilitated the understanding of the dynamics of HIV infection and the interaction of the immune system with the virus. Much work has been done using ordinary differential equation (ODE) and partial differential equation (PDE) models (Arnaout et al., 2000; De Boer and Perelson, 1989; Dixit and Perelson, 2005; Kirschner, 1996; Wodarz and Nowak, 1998). These models have explained different aspects of the dynamics of the virus-immune system interaction. However, because HIV infection typically exhibits a three-phase evolution (acute phase, chronic phase, and AIDS) (Pantaleo and Fauci, 1996), these continuous deterministic system models may be insufficient to describe the different time scales (days, weeks, and years) that are involved. Furthermore, an additional drug treatment response phase is even

\footnotetext{
*Corresponding author. Tel.: + 14809656915 ; fax: + 14809658119.

E-mail address: kuang@asu.edu (Y. Kuang).
}

more difficult to simulate using these models, not to mention the different types of therapy and the corresponding responses.

Recently, cellular automata (CA) models have been used in HIV modeling (Sloot et al., 2002; Zorzenon dos Santos and Coutinho, 2001) and have shown great potential for simulating the temporal and spatial immune responses throughout the course of HIV infection. The strength of the CA model lies in its simplicity and, at the same time, its ability to model complex systems (Ganguly et al., 2003). CA models also allow for more granularity of the simulation, which is hard to do with ODE and PDE models.

CA were first used by Zorzenon dos Santos and Coutinho (2001) to model the evolution of HIV infection. A set of four simple rules was adopted to represent the life cycles of the $C D 4^{+} \mathrm{T}$ cells in terms of four states, namely healthy (T), infected stage 1 (A1), where the cell is infected and able to spread the infection, infected stage 2 (A2), the final stage of an infected cell before it dies, and dead (D). It has been proposed that the lymphoid tissue is the primary 
target and major reservoir of HIV infection (Fauci, 2003) in vivo, and a lymph node has a mesh structure that may be approximated by a rough surface (Hood et al., 1984). Therefore, it is reasonable to model the interaction among the immune system cells in the lymphoid tissue using square lattice. The basic dos Santos model produced results that qualitatively matched the three-phase HIV dynamics observed from clinical data. Although their work presented a novel approach to the modeling of HIV dynamics, critics raised issues concerning the model's sensitivity to parameters (Strain and Levine, 2002). One particular issue was that the initial density of infected cells, $P_{H I V}=0.05$, was too large compared to the estimated value from clinical experiments. When $P_{H I V}$ was set to less than 0.05 , the initial infection peak did not occur in the model, and there were no distinct first-phase dynamics.

Based on Zorzenon dos Santos' CA model, Sloot et al. (2002) developed a drug therapy CA model by modifying one of the rules. Instead of infecting all eight neighbors of an infected cell, the number of neighbors to be infected was set to $N(0 \leqslant N \leqslant 7)$ with probability $P_{\text {resp }}$, and 8 with probability $\left(1-P_{\text {resp }}\right)$. The number $N$ was used to mimic the drug effectiveness, i.e., the smaller the $N$, the more efficient the drug. The probability $P_{\text {resp }}$ represented the patient's response to the therapy: the higher the probability, the better the treatment. Drug resistance was modeled by using a linearly decreasing $P_{\text {resp }}$.

The weakness of this approach is multifold: (1) there is no quantitative and direct association of the number of neighbors to be infected to the efficacy of the drug; (2) the number of neighbors to be infected is predefined before the simulation run and fixed throughout the simulation run, which does not change with the progression of the disease; and (3) the model is not based on the principles of drug treatment, and therefore, there is no differentiation between different types of drug treatments, such as a protease inhibitor or a reverse transcriptase (RT) inhibitor. One important factor that was omitted in both of the models by Sloot et al. (2002) and Zorzenon dos Santos and Coutinho (2001) was the effect of viral load on the dynamics of the infection and drug treatment. We will show later in this paper that our model has addressed all these and many other important aspects.

Here we describe a simple, flexible, yet comprehensive and practically useful CA model of HIV dynamics that is built upon realistic biological processes. The model is based on biological processes of virus replication, the principles of drug treatment, and the different stages of the $C D 4^{+} \mathrm{T}$ cell as the immune system responds (Ho, 1997; Nowak et al., 1996; Nowak and May, 2000; Perelson et al., 1993, 1996). To produce a model with biological fidelity, many aspects of the infection dynamics were incorporated, including the following: the fact that HIV is a retrovirus and its replication is through translation of viral RNA into the host cell's DNA by RT; that the latently and chronically infected $C D 4^{+} \mathrm{T}$ cells play an important role in sustaining viral replication and contributing to the eventual sharp rise in viral levels and collapse of the immune system; that the viral load is closely correlated to the progression of the disease; and that the basic principle of drug therapy is to disrupt virus replication, either through protease or RT inhibition. Once this model is tested and verified, it can be used to further understand this disease and help with the evaluation of different treatment strategies.

\section{Model development}

Zorzenon dos Santos' CA model (Zorzenon dos Santos and Coutinho, 2001), which will be referred to as the basic CA model throughout the rest of the paper, serves as the starting point of the model development. New cell states and new rules are adopted to include many aspects of the viral dynamics and disease evolution.

\subsection{Modeling latently infected cells}

Recent studies have shown that a pool of latently infected cells is established during the primary phase of HIV infection (Bonhoeffer et al., 1997; Grossman et al., 1998; Havlir et al., 2003; Lafeuillade et al., 2000; Muller et al., 2002). These cells are infected cells in a resting state and can be activated after a long time of dormancy to produce infectious virus particles. The reservoirs of these dormant infected cells play a critical role in sustaining active infection (Bailey et al., 2004; Ramratnam et al., 2000). The process of the activation and transmission of these long-lived latently infected cells is the most plausible explanation of HIV propagation during the chronic phase.

To incorporate this important aspect of HIV dynamics, a new cell state is added - the latently infected state (A0). When a cell is to become infected based on the original rules, an additional rule is used to decide whether a cell should go into the actively infected (A1) state with a probability $P_{\text {inf }}$ or the latently infected (A0) state with a probability $\left(1-P_{\text {inf }}\right)$. After a long time delay, the latently infected cell may become activated with a probability $P_{a c t}$.

\subsection{Viral load}

The basic class of ODE HIV models (Culshaw and Ruan, 2000; Nelson and Perelson, 2002; Nelson et al., 2000; Perelson and Nelson, 1999) generally includes variables that represent the healthy cells $\mathrm{T}$, infected cells $T^{*}$, and the viral load $V$. The viral load is an integral part of the system of equations that influences the dynamics of the healthy and infected cells.

Conventional CA models are built with healthy and infected cell states. Rules control the state transitions; therefore, the models autonomously account for the dynamics of the healthy and infected cells. However, none of the CA models of previous studies included the viral load. The previous assumption was that the viral load was simply proportional to the number of infected cells, effectively ignoring the role of free virions in the 
progression of the disease. To account for this important factor in the dynamics of the disease, a novel approach is developed to include the effect of viral load on the infection rate in the $\mathrm{CA}$ model.

A difference equation can be written as shown below to describe the dynamics of the viral load, assuming the time interval $\Delta t=1$

$\Delta V_{t}=\delta T_{t}^{*}-c V_{t}, \quad V_{t+1}=\delta T_{t}^{*}+(1-c) V_{t}$,

where $\delta$ is the average virion production rate per infected cell and $c$ is the virion decay rate.

To incorporate the viral load into the CA model, Eq. (1) is calculated at each time step after all the cells in the lattice have been updated according to the rules. The viral load is then used to adjust the number of cells in the domain of the neighborhood that will affect the state of the center cell in the next time step. This effectively builds a feedback loop, which affects the infection rate at each time step. The following sections detail how the viral load is incorporated into the definition of neighborhood domain and its effect on infection rate.

\subsection{Viral load and expanded neighborhood}

The conventional CA models in previous studies all use a neighborhood of eight cells where the rules are applied to determine changes in cell state. In CA models, the effect of the number of neighbors used is equivalent to the infection rate since it determines how fast the infection spreads. Because previous studies used constant 8-cell neighborhoods, this meant that the infection rate did not change in those models. A more realistic simulation would be to adjust the number of cells in the neighborhood based on the viral load. The biological mechanism of the HIV infection indicates that healthy $\mathrm{T}$ cells become infected in the presence of the HIV virus. The higher the virus concentration, the higher the probability of infection. In order to allow for the modeling of a higher infection rate, the domain of the neighborhood must be allowed to expand beyond the eight adjacent neighbors. Based on the Moore neighborhood definition (Weisstein), the neighborhood of range $r$ is defined by

$N_{\left(x_{0}, y_{0}\right)}^{M}=\left\{(x, y):\left|x-x_{0}\right| \leqslant r,\left|y-y_{0}\right| \leqslant r\right\}$.

The number of cells in the Moore neighborhood of range $r$ is the odd square $(2 r+1)^{2}$. When the center cell is excluded from the neighborhood, as in the CA model, the number of neighbors is $(2 r+1)^{2}-1$. For the 8-cell neighborhood the corresponding range $r$ is 1 . A new concept is to extend the definition of range $r$ beyond the integer domain to include real numbers, such that the neighborhood is still defined in the same way. For example, a range $r$ of 1.5 corresponds to a 15-cell neighborhood.

The range $r$ can be considered the equivalent of the range of infection, i.e., a healthy cell may be infected by an infected cell within the neighborhood of radius $r$. Biologically, long-range transmission of the infection is possible under high viral burden (Nowak and May, 2000). Allowing the number of cells in the neighborhood to vary with the amount of viral load allows the modeling of changes in infection rate, which leads to a more realistic model and a flexible way of incorporating different types of drug treatment.

In order to include the viral load in the CA model, we need to find the relationship between the viral load and the number of cells in the neighborhood. Intuitively, the number of neighbors is proportional to viral load, because the higher the viral load, the more infectious it is, and thus the more possibilities for long-range infection. However, because of the discrete nature of the automata and the rules governing the infection of the healthy cells, the translation from viral load to the number of neighbors is more complicated than a simple linear or exponential function.

In all the previous studies, cell state change had been based on the cell states of the 8-cell neighborhood and a set of rules. Rule 1 of the basic CA model dictates that if one of the eight neighbors of a cell is infected, then the cell becomes infected. This rule governs how the number of infected cells around an original infected cell grows, which is in the order of $1,9,25,49,81$, etc. as time goes on. Table 1 summarizes how the number of infected cells grows for a Moore neighborhood range of 1 and 2 .

Examining Table 1 leads to a generic formula for $r=1$ :

$\Delta T_{n}^{*}=T_{n}^{*}-T_{n-1}^{*}=n \cdot M_{1} \cdot T_{0}^{*} \quad$ and $\quad M_{1}=\frac{\Delta T_{n}^{*}}{n \cdot T_{0}^{*}}$.

Further examination of Table 1 for $r=2$ reveals that the coefficients of $\Delta T_{n}^{*}$ form an arithmetic series in the form of $a_{n}=a_{1}+(n-1) d$, where $d=1$ and $a_{1}=1$ for $r=1$, and $d=4$ and $a_{1}=3$ for $r=2$, relative to $M_{1}=8$, i.e., $M_{2}=24=3 \cdot M_{1}=3 \cdot \Delta T_{n}^{*} / a_{n} \cdot T_{0}^{*}$.

Table 1

Growth of number of infected cells around an original infected cell

\begin{tabular}{llll}
\hline Time step, $t$ & $r=1$ & & $r=2$ \\
\cline { 2 - 3 } & Number of $T^{*}$ & $\Delta T^{*}$ & Number of $T^{*}$ \\
\hline 1 & $(8+1) \cdot T_{0}^{*}$ & $8 \cdot T_{0}^{*}=M_{1} \cdot T_{0}^{*}$ & $(24+1) \cdot T_{0}^{*}$ \\
2 & $(24+1) \cdot T_{0}^{*}$ & $16 \cdot T_{0}^{*}=2 \cdot M_{1} \cdot T_{0}^{*}$ & $(80+1) \cdot T_{0}^{*}$ \\
3 & $(48+1) \cdot T_{0}^{*}$ & $24 \cdot T_{0}^{*}=3 \cdot M_{1} \cdot T_{0}^{*}$ & $(168+1) \cdot T_{0}^{*}$ \\
4 & $(80+1) \cdot T_{0}^{*}$ & $32 \cdot T_{0}^{*}=4 \cdot M_{1} \cdot T_{0}^{*}=3 \cdot M_{1} \cdot T_{0}^{*}$ & $(288+1) \cdot T_{0}^{*}=7 \cdot M_{1} \cdot T_{0}^{*}$ \\
& & $88 \cdot T_{0}^{*}=11 \cdot M_{1} \cdot T_{0}^{*}$ & $120 \cdot T_{0}^{*}=15 \cdot M_{1} \cdot T_{0}^{*}$
\end{tabular}


Therefore Eq. (3) can be generalized as

$M_{r}=s \cdot \frac{\Delta T_{n}^{*}}{a_{n} \cdot T_{0}^{*}}$.

Rule 2 of the basic CA model also specifies that an infected-A1 cell becomes infected-A2 after $\tau$ time steps, where $\tau=4$. The infected-A2 cells die in the next time step according to rule 3 . Therefore, for $r=1$ we have

$T_{5}^{*}=(120+1) \cdot T_{0}^{*}-T_{0}^{*}=15 \cdot M_{1} \cdot T_{0}^{*}$,

$T_{6}^{*}=(168+1) \cdot T_{0}^{*}-(8+1) \cdot T_{0}^{*}=20 \cdot M_{1} \cdot T_{0}^{*}$

and

$\Delta T_{6}^{*}=T_{6}^{*}-T_{5}^{*}=5 \cdot M_{1} \cdot T_{0}^{*}$

and Eq. (4) becomes

$M_{r}=s \cdot \frac{\Delta T_{n}^{*}}{a_{n} \cdot \Delta T_{0}^{*}}, \quad \begin{cases}a_{n}=a_{1}+(n-1) \cdot d, & n<\tau, \\ a_{n}=5 \cdot r^{2}, & n \geqslant \tau .\end{cases}$

Now assuming that the viral load $V$ is strictly proportional to the number of infected cells $T^{*}$ (i.e., the decay of the virion can be ignored for the moment), then the number of neighbors $M_{r}$ can be expressed as

$M_{r}=s \cdot \frac{\Delta T_{n}^{*}}{a_{n} \cdot T_{0}^{*}}=s \cdot \frac{\delta \cdot \Delta T_{n}^{*}}{a_{n} \cdot \delta T_{0}^{*}}=s \cdot \frac{\Delta V_{n}}{a_{n} \cdot \delta T_{0}^{*}}$.

Although Eq. (6) is derived based on the expansion of the infected cells, it is assumed that it still holds true during the declining phase of the infection. Biologically, the infection rate is not only related to the amount of virions in the system, but also to the spatial distribution of the virion concentration. In other words, the growth rate of the infected cells is proportional to the peaks of the local concentration of virions, not to the average concentration throughout the system. Eq. (6) indicates that the number of neighbors is related to the change of the viral load, which is consistent with the biological characteristics. Without an elaborate algorithm to figure out the geometric distribution of the infected cell clusters, we will make a simple approximation by assuming that the change of number of neighbors, $\Delta M$, is a fraction of $M$ and is proportional to the change of the total viral load: $\Delta M=M / h=(s / h) \cdot \Delta V_{n} / a_{n} \delta T_{0}^{*}$. Then by choosing a proper coefficient $h$ and combining $h$ with the scale factors $s$ and $a_{n}, 1 / k=s / h a_{n}$, and we have

$\Delta M_{t}=\frac{V_{t}-V_{t-1}}{k \delta T_{0}^{*}}, \quad M_{t+1}=M_{t}+\Delta M_{t}$.

\subsection{Drug treatment}

With the advances in medicine, there are many drugs available to treat patients with HIV. Currently, two types of basic inhibitors are used-RT inhibitors and protease inhibitors. The RT inhibitors block the RT process of the virus after it enters a healthy cell, preventing the cell from being infected. The protease inhibitors cause infected cells to produce non-infectious virions. Standard mono-therapy uses RT inhibitors alone. Combination therapy of RT inhibitors with later-developed protease inhibitors usually yields better treatment. The latest development in drug therapy is the highly active antiretroviral therapy (HAART), which typically combines three or more drugs of both the RT and protease inhibitors.

To simulate the effect of the RT inhibitor, a new cell state, the exposed but not infected state (An), is added to the model to represent the state where a cell is invaded by the virus but stays healthy because of the treatment. The efficacy of the drug is simulated by a probability $P_{r t}$ that decides whether a healthy cell, normally to be infected under the rules when no treatment is given, will be infected (A1) or stay in an exposed but not infected (An) state due to RT inhibition. The An cells will remain uninfected for a short period of time before they become susceptible to infection again, either due to viral RNA degradation or new strains of viral mutation.

Assume the efficacy of the protease inhibitor is represented by the numerical value of the percentage $P_{p i}$ of virions that are non-infectious during burst. Then the simulation of the protease inhibitor is a simple change of the viral load equation (1):

$V_{t+1}=\left(1-P_{p i}\right) \cdot \delta T_{t}^{*}+(1-c) \cdot V_{t}$.

Because the viral load affects the infection rate through the change of the number of neighbors, the CA model will autonomously adjust the dynamics of the infection to reflect the effect of the protease inhibitor therapy.

Combination therapy and HAART can be modeled by setting both $P_{r t}$ and $P_{p i}$ to levels that represent the efficacy of the individual RT and protease inhibitors or the combined efficacy of two or more RT or protease inhibitors.

Drug resistance is modeled by using a variable diminishing drug efficacy $P_{r t}$ or $P_{p i}$. One possible form of the declining efficacy is to assume a decay model of $P_{r t}^{\prime}=-l P_{r t}$. Solving the differential equation gives $P_{r t}=P_{r t 0} \cdot \mathrm{e}^{-l\left(\left(t-t_{s}\right) / t_{s}\right)}$, where $t_{s}$ is the drug therapy start time.

\section{Methods}

A square lattice with $700 \times 700$ cells was used to simulate the lymphoid tissues. The lattice was initialized with infected cells randomly distributed among healthy cells. Each time step represented 1 week. At each time step, the state of each cell in the lattice was updated based on the rules and its neighbors' states. After all the cell states were updated, the viral load was calculated using Eq. (1), and the number of cells in the neighborhood that would affect the state of the center cell was updated for the next time step using Eq. (7).

The CA model was implemented in MATLAB. Configurations were set up for different simulation purposes, such as no treatment, mono or combination therapy, and 
HAART treatment. For each configuration, multiple simulation runs were conducted and the data averaged to produce the final results. The following sections outline the details of the CA model, including cell states, rules, and parameters used.

In this model, there are six cell states as opposed to the four states that Zorzenon dos Santos and Coutinho (2001) and Sloot et al. (2002) used. In addition to the healthy (T), infected stage 1 (A1), infected stage 2 (A2), and dead (D) states, the latently infected (A0) and exposed but not infected $(\mathrm{An})$ states are incorporated to better simulate the biological processes of HIV infection. A state transition diagram is presented in Fig. 1 to illustrate the relationship of these states and the conditions for each state transition.

The states of all cells are updated using the rules listed in Table 2 at each time step. Viral load and the number of cells in the neighborhood are updated after all the cells are updated. Each time step represents 1 week.

Table 3 lists all the parameters used in the model. The unit for time is in weeks.

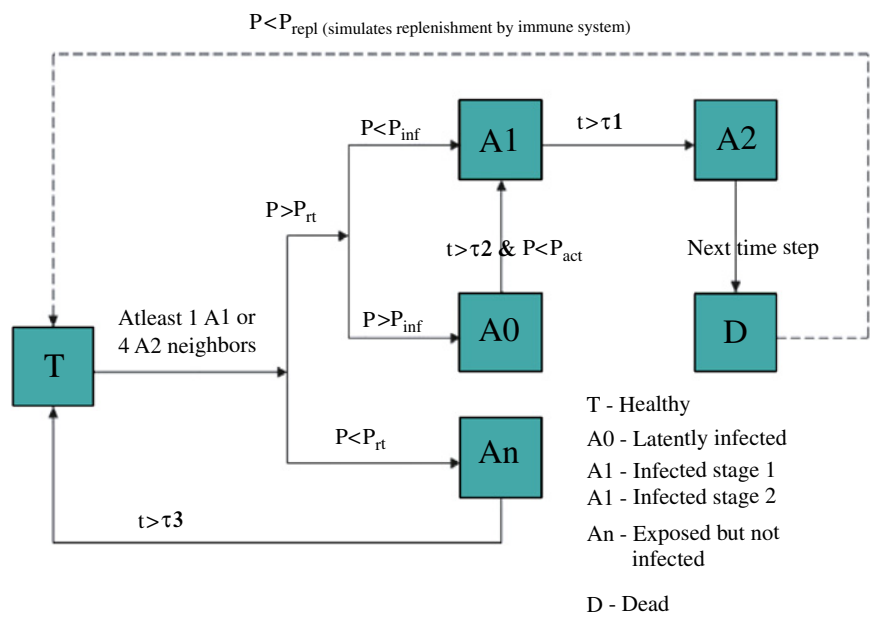

Fig. 1. State transition diagram of cell states.

\section{Results}

The CA model developed here is a comprehensive tool that can simulate different drug therapy regimens. By setting the parameters $P_{p i}$ and $P_{r t}$ to different values to form various configurations, different types of treatment can be evaluated. Because each simulation run represents a particular individual case, the simulation was run 20 times for each configuration and the results averaged over the runs. The following figures show the results from the simulation runs of selected configurations.

When $P_{p i}$ and $P_{r t}$ are set to zero, the model is simulating the effect of no drug treatment. Fig. 2 shows the dynamics of the HIV infection without treatment. The three phases of the infection, acute, chronic, and AIDS, are evident in the graph, which shows that the model output qualitatively matches clinical data. Note the different time scales of weeks and years in the graphs. It is also worth mentioning that the probability that determines the number of initial infected cells $P_{H I V}$ is set to 0.005 as opposed to 0.05 in the dos Santos' model. The value of 0.05 , according to critics, was too high compared to clinical experiment data, and the dos Santos' model failed to show the acute phase dynamics when $P_{H I V}$ was set to 0.005 (Strain and Levine, 2002). By incorporating the viral load and varying the number of cells in the neighborhood, the infection rate changes based on viral load, and the model is able to maintain the acute phase characteristics even when $P_{H I V}$ equals 0.005 . The results presented here prove the robustness of this model. The graphs also show that the number of cells in the neighborhood truthfully reflects the viral load and effectively changes the infection rate. Both the viral load and M-cell neighbors exhibit the three-phase dynamics. The results also show that the chronic phase is sustained by the activation of the latently infected cells.

In addition to simulating the dynamics of the HIV infection without treatment, the model is very flexible in simulating drug therapy. Different types of drug therapy

Table 2

Model rules

\begin{tabular}{|c|c|}
\hline Rule no. & Rule description \\
\hline \multirow[t]{2}{*}{ Rule 1} & Update of healthy cells \\
\hline & $\begin{array}{l}\text { If a healthy cell has at least one A } 1 \text { neighbor or } R \text { A } 2 \text { neighbors within the M-cell neighborhood, then it becomes an An cell with } \\
\text { probability } P_{r t} \text {. Otherwise it becomes an infected A } 1 \text { cell with probability } P_{\text {inf }} \text { or a latently infected A } 0 \text { with probability }\left(1-P_{\text {inf }}\right) \text {. } \\
\text { Otherwise it stays healthy. }\end{array}$ \\
\hline \multirow[t]{2}{*}{ Rule 2} & Update of infected-A1 cells \\
\hline & An infected-A1 cell becomes infected-A2 cell after $\tau 1$ time steps. \\
\hline \multirow[t]{2}{*}{ Rule 3} & Update of infected-A2 cells \\
\hline & Infected-A 2 cells become dead cells in the next time step. \\
\hline \multirow[t]{2}{*}{ Rule 4} & Update of dead cells \\
\hline & $\begin{array}{l}\text { Dead cells can be replaced by healthy cells with probability } P_{\text {repl }} \text { in the next time step (or remain dead with probability } 1-P_{\text {repl }} \text { ). This } \\
\text { simulates the new cells that are replenished by the immune system. }\end{array}$ \\
\hline \multirow[t]{2}{*}{ Rule 5} & Update of latently infected A0 cells \\
\hline & After a long time delay $(\tau 2)$, the latently infected-A0 cell becomes actively infected (A1) with a probability $P_{a c t}$, otherwise it stays A0 \\
\hline \multirow[t]{2}{*}{ Rule 6} & Update of exposed but not infected An cells \\
\hline & After a short time delay $(\tau 3)$, the An cells become healthy $(\mathrm{T})$ cells. \\
\hline
\end{tabular}


Table 3

Model parameters

\begin{tabular}{|c|c|c|c|}
\hline Parameter & Description & Value & Source \\
\hline$L$ & Lattice size, total of cells in the lattice is $L^{*} L$ & 700 & Zorzenon dos Santos and Coutinho (2001) \\
\hline$P_{H I V}$ & Probability or percentage of initial infected cells & 0.005 & $\begin{array}{l}\text { Sloot et al. (2002), Strain and Levine (2002), } \\
\text { Zorzenon dos Santos and Coutinho (2001) }\end{array}$ \\
\hline$P_{\text {inf }}$ & $\begin{array}{l}\text { Probability that a cell becomes actively infected, } \\
\text { probability for latently infected is }\left(1-P_{\text {inf }}\right)\end{array}$ & 0.999 & ad hoc \\
\hline$P_{\text {act }}$ & Probability that a latently infected cell is activated & 0.0005 & ad hoc \\
\hline$P_{\text {repl }}$ & Probability of replacing dead cell with healthy cell & 0.99 & Zorzenon dos Santos and Coutinho (2001) \\
\hline$P_{p i}$ & Drug efficacy of protease inhibitor & $50-90 \%$ & Variable \\
\hline$P_{r t}$ & Drug efficacy of reverse transcriptase inhibitor & $50-90 \%$ & Variable \\
\hline$R$ & $\begin{array}{l}\text { Number of A2 cells in the neighborhood to cause the } \\
\text { center cell to become infected }\end{array}$ & 4 & Zorzenon dos Santos and Coutinho (2001) \\
\hline$\tau 1$ & Time delay for an A1 cell to become an A2 cell & 4 & Zorzenon dos Santos and Coutinho (2001) \\
\hline$\tau 2$ & Time delay during which A0 cells stay inactive & 30 & ad hoc \\
\hline$\tau 3$ & $\begin{array}{l}\text { Time delay before an An cell } \\
\text { becomes susceptible to infection again }\end{array}$ & 1 & ad hoc \\
\hline$c$ & Virion decay rate & 0.3 & Based on Nelson et al. (2000) \\
\hline$k$ & $\begin{array}{l}\text { Coefficient to convert viral load to number of cells in the } \\
\text { neighborhood }\end{array}$ & $\begin{array}{l}30, \text { if } \\
T_{t}^{*} \geqslant T_{0}^{*} ; 12, \text { if } \\
T_{t}^{*}<T_{0}^{*}\end{array}$ & ad hoc \\
\hline$\delta$ & Average virion production rate per infected cell & 480 & Nelson et al. (2000) \\
\hline
\end{tabular}
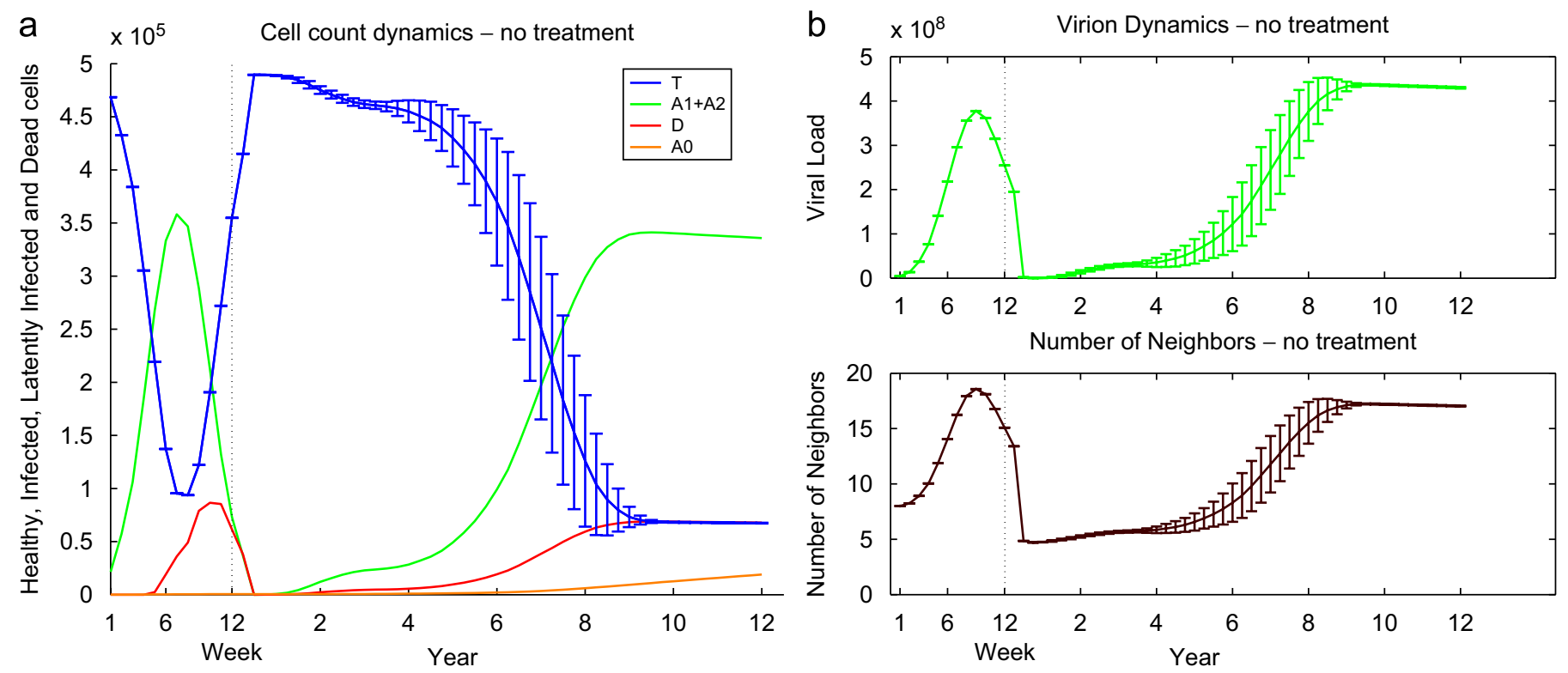

Fig. 2. HIV dynamics without drug treatment. (a) Plots of healthy cells (T), infected cells (A1 + A2), dead cells (D) and latently infected cells (A0). The standard error of the mean (SEM) for healthy cells is shown with the other SEMs omitted to avoid crowding. (b) Graphs of the viral load and the number of cells in the neighborhood over time. This shows that the neighborhood size, and thus the infection rate, is indeed based on the viral load in the model.

and dosages are modeled by setting different $P_{p i}$ and $P_{r t}$ values. Protease inhibitor treatment is modeled by setting $P_{r t}$ to zero and $P_{p i}$ to certain values, and similarly, RT inhibitor treatment is modeled by setting $P_{p i}$ to zero and $P_{r t}$ to certain values. Fig. 3 shows the effects of the protease inhibitor and RT inhibitor drug treatment schemes.

The results show that there is a sharp response to the drug treatment characterized by the rise in the number of healthy $\mathrm{T}$ cells and decrease in viral load and the infection rate. The response curves are all in order of the drug efficacy, i.e., a higher efficacy produces a more prominent response.

The graphs also demonstrate that treatment with the RT inhibitor shows a better prognosis. One possible explanation is that although the protease inhibitor reduces the number of virions that each infected cell produces, the 

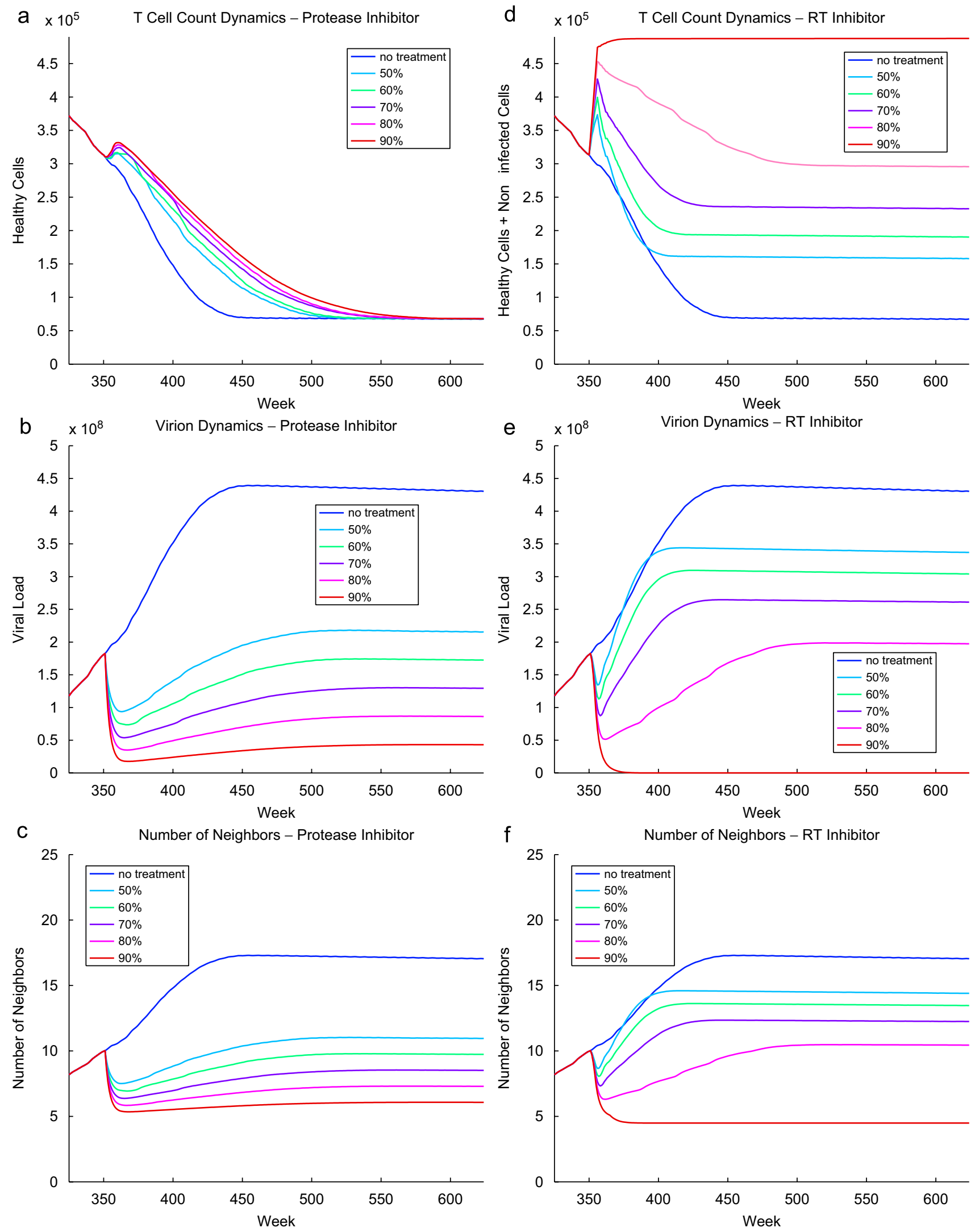

Fig. 3. Dynamics of the HIV infection with protease inhibitor and reverse transcriptase inhibitor treatment, with graphs of the level of T cells, the viral load, and the number of neighbors. (a)-(c) Drug treatment with protease inhibitor. (d)-(f) RT inhibitor treatment. Each curve represents one drug efficacy, which ranges from $50 \%$ to $90 \%$. Therapy starts at week 350 . 
effect on the spread of the disease is less direct than that of the RT inhibitor, which prevents the cells from being infected for a period of time. At later stages of the disease where the number of infected cells are high, merely reducing the replication of the virus from each infected cell may not have enough aggregative effect. In the CA model of Sloot et al. (2002), the reduction of the number of neighbors had to be explicitly specified along with a response function in order to simulate the drug treatment. In this model, only the drug efficacy $P_{r t}$ needs to be specified. Because the model incorporates the effect of viral load on infection rate, the number of neighbors is automatically adjusted once the drug is in effect. This effect can be seen clearly in the graphs. This model also makes clear distinctions between the sources of inhibition, and the different effects can be analyzed using the simulation results, whereas in the Sloot model there is no difference between the RT and protease inhibitors.

Combination therapy or HAART can be modeled by setting different combinations of $P_{r t}$ and $P_{p i}$. For example, a combination therapy may consist of one protease inhibitor drug with an efficacy of $70 \%$ and one RT inhibitor with an efficacy of $80 \%$. Similarly, it could be a combination of one protease inhibitor and two RT inhibitors with a combined efficacy of $90 \%$.

The results from a few selected configurations are shown in Fig. 4. Compared with the results from the simulations of mono-therapy (Fig. 3a and d), the improvement with a combined treatment is obvious. This matches the general consensus from a theoretical standpoint, as well as from clinical trials, that treatments with combined antiviral drugs provide more favorable outcome than with monotherapy regimens (Hammer et al., 1996; Kirschner and Webb, 1998).

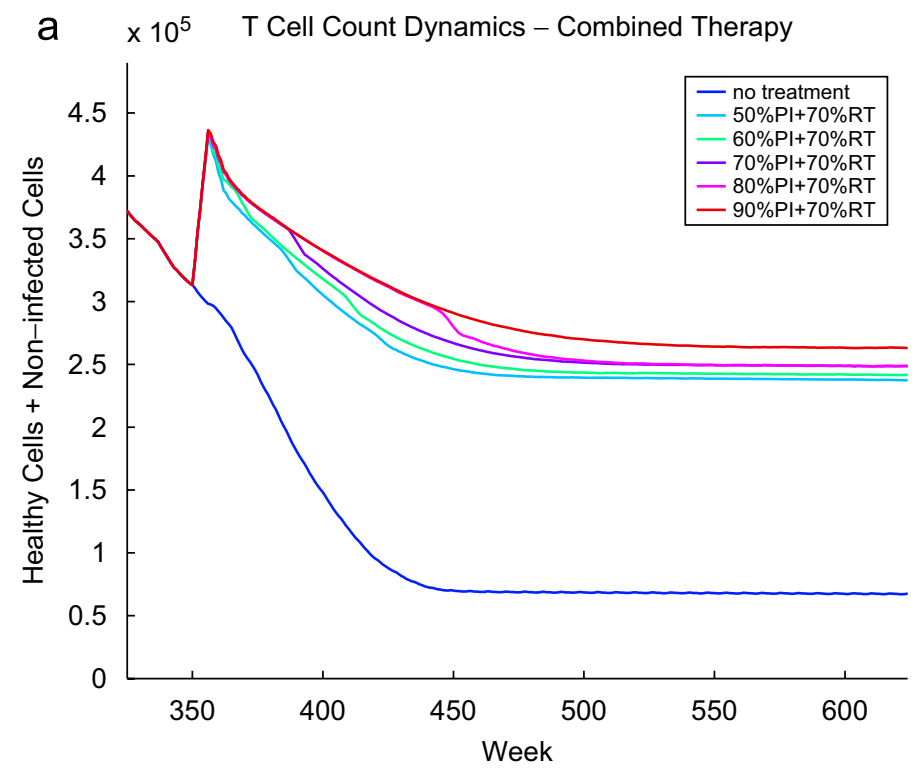

To model drug resistance, a variably diminishing drug efficacy $P_{r t}$ is used, where $P_{r t}=P_{r t 0} \cdot \mathrm{e}^{-((t-350) / 350)}$, and the results are shown in Fig. 5. Graph 5a shows mono-therapy with the RT inhibitor and 5b shows combination therapy with both the protease and RT inhibitors. Comparing graphs $5 \mathrm{a}$ with $3 \mathrm{~d}$ and $5 \mathrm{~b}$ with $4 \mathrm{~b}$, it is evident that the effectiveness of the drug treatment drops as resistance builds up.

In order to model dosage adjustment and therapy scheduling, the viral load and healthy count thresholds and their corresponding drug efficacies were empirically determined and are shown in Table 4. The dosage change allowed at each check-up time was limited to the equivalent of a change in drug efficacy of 0.1 .

The results of this particular approach of dosage adjustment and scheduling are shown in Fig. 6. It is evident that the drug dosage starts low and gradually increases as the infection progresses. The dosage levels, along with the viral load and healthy cell counts are finally stabilized, indicating that the disease is under control. Notice that the efficacies of both the protease inhibitor $P_{p i}$ and RT inhibitor $P_{r t}$ are below the maximum when stability is achieved $\left(P_{p i}=0.8\right.$ and $\left.P_{r t}=0.7\right)$, indicating a good balance of drug effectiveness and toxicity level, leading to a better chance of therapy adherence by the patient.

To quantitatively evaluate the effectiveness of the different drug treatment schemes, the results were further analyzed based on the stages of HIV infection. Clinical diagnosis often categorizes the disease progression into stages based on immune monitoring tests, such as a viral load test. The main stages are the primary infection, asymptomatic and symptomatic stages, with the symptomatic stage further broken down into early, medium, and

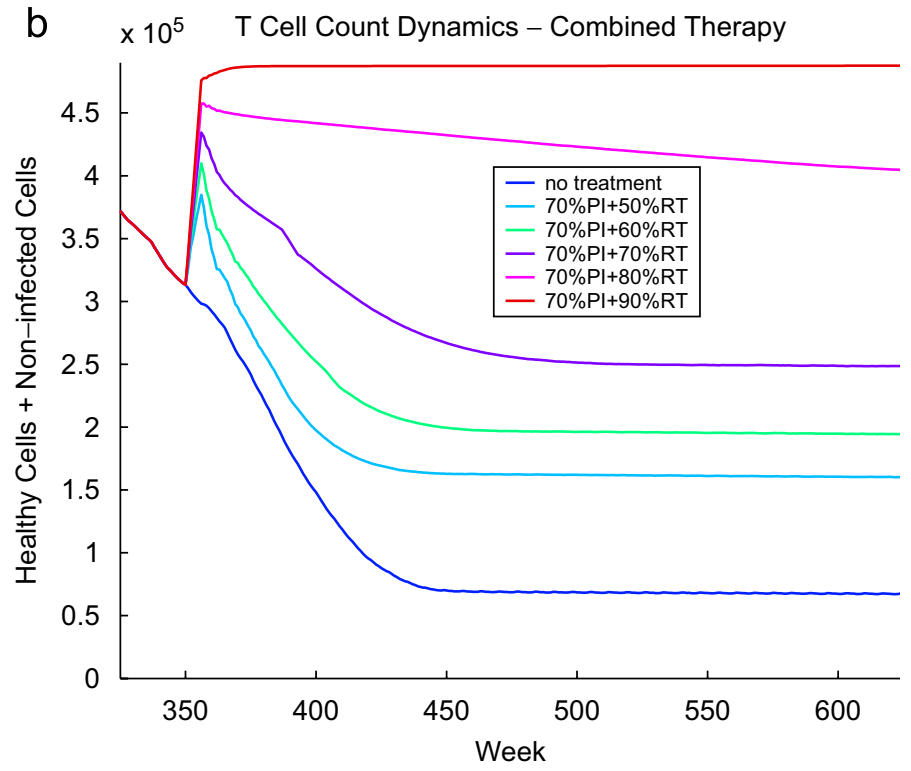

Fig. 4. Results from simulations of combination therapy or HAART therapy, showing the level of T cells. (a) Efficacy of the RT inhibitor is $70 \%$ for all curves in the graph, protease inhibitor efficacy ranges from $50 \%$ to $90 \%$. (b) Efficacy of the protease inhibitor is $70 \%$ for all curves, RT efficacy ranges from $50 \%$ to $90 \%$. 

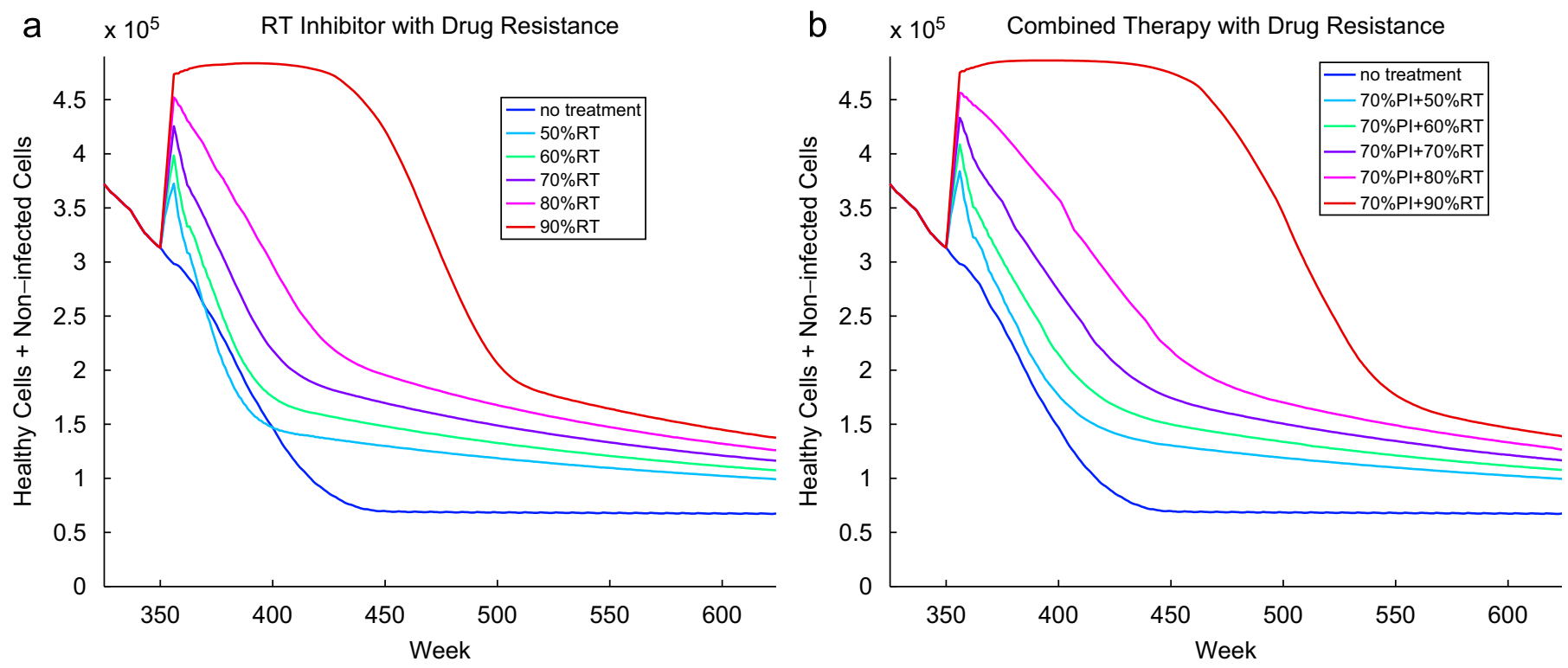

Fig. 5. Simulation results with drug resistance showing the level of healthy cells. (a) RT inhibitor only. (b) RT inhibitor plus protease inhibitor.

Table 4

Dosage adjustment and scheduling

\begin{tabular}{lllll}
\hline $\begin{array}{l}\text { Viral load threshold } \\
\left(\times 10^{8}\right)\end{array}$ & $\begin{array}{l}\text { Protease inhibitor efficacy } \\
\left(P_{p i}\right)\end{array}$ & $\begin{array}{l}\text { Check-up interval } \\
(\text { weeks, based on } V \text { only })\end{array}$ & $\begin{array}{l}\text { Healthy cell count threshold } \\
(\mathrm{T}+\mathrm{An})\left(\times 10^{5}\right)\end{array}$ & $\begin{array}{l}\text { Reverse transcriptase } \\
\text { inhibitor efficacy }\left(P_{r t}\right)\end{array}$ \\
\hline$V \leqslant 0.3$ & $\begin{array}{l}\text { No treatment } \\
\left(P_{p i}=0\right)\end{array}$ & 8 & $(\mathrm{~T}+\mathrm{An}) \leqslant 1.25$ & 0.95 \\
$0.3<V \leqslant 0.8$ & 0.4 & 8 & $1.25<(\mathrm{T}+\mathrm{An}) \leqslant 1.75$ & 0.9 \\
$0.8<V \leqslant 1.3$ & 0.5 & 6 & $1.75<(\mathrm{T}+\mathrm{An}) \leqslant 2.25$ & 0.8 \\
$1.3<V \leqslant 1.8$ & 0.6 & 6 & $2.25<(\mathrm{T}+\mathrm{An}) \leqslant 2.75$ & 0.7 \\
$1.8<V \leqslant 2.3$ & 0.7 & 4 & $2.75<(\mathrm{T}+\mathrm{An}) \leqslant 3.25$ & 0.6 \\
$2.3<V \leqslant 2.8$ & 0.8 & 4 & $3.25<(\mathrm{T}+\mathrm{An}) \leqslant 3.75$ & 0.5 \\
$2.8<V \leqslant 3.3$ & 0.9 & 4 & $3.75<(\mathrm{T}+\mathrm{An}) \leqslant 4.25$ & 0.4 \\
$V>3.3$ & 0.95 & 2 & $(\mathrm{~T}+\mathrm{An})>4.25$ & No treatment \\
& & & & $\left(P_{r t}=0\right)$ \\
\hline
\end{tabular}

late symptomatic stages. Analogous to the process of clinical diagnosis, the model results are tabulated into stages based on viral load for each week. From the histograms of the occurrences of the stages, the effectiveness of the drug treatment can be readily seen.

Fig. 7 shows the frequency of the stages for some selected treatment schemes. The results show that when no drug treatment is given, the number of weeks in the asymptomatic stage is relatively low and the number of weeks in the late symptomatic stage is relatively high. The effectiveness of the drug treatment increases from graphs $7 \mathrm{~b}$ to $7 \mathrm{~d}$ with the graph showing the combined therapy of PI and RTI both at $90 \%$ having the highest duration of the asymptomatic stage and the lowest duration of the late symptomatic stage. Note that drug resistance modeling was included in these results, so the effectiveness of the therapy would be more prominent if there was no resistance.

\section{Discussion}

The CA model presented here successfully simulates the three distinctive phases of the HIV infection with no treatment and an additional drug response phase when treatment is applied. The key new feature of this CA model is that the number of cells in the neighborhood which influences the center cell's state is calculated based on the viral load. This variable-cell neighborhood enables the simulation of an infection rate that is correlated to the viral load. The inclusion of these free virions and their decay, and the effect on infection rate, gives this model a more realistic biological foundation. The introduction of this novel concept of a variable-cell neighborhood not only leads to a whole new method of constructing CA models, but also allows for flexibility in modeling HIV dynamics. In addition, this approach enables the modeling of different antiretroviral drug treatments to closely reflect 

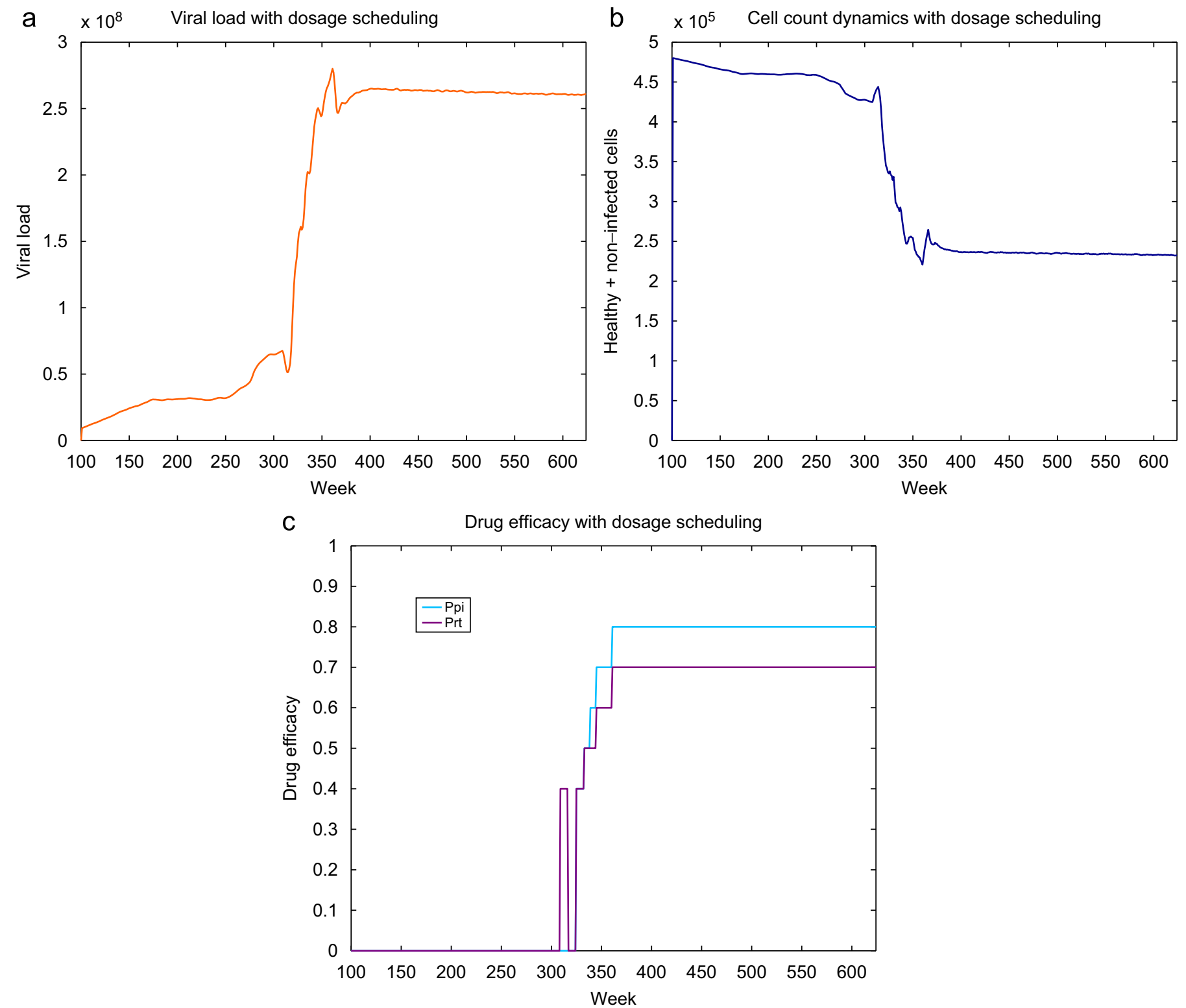

Fig. 6. Dosage adjustment and scheduling. (a) Graph of viral load levels over time. (b) Graph of healthy cell count over time. (c) Graph of drug efficacies over time.

their individual and combined biological effects. The protease inhibitor treatment is simulated by reducing the number of infectious virions produced by infected cells, and the RT inhibitor is modeled by cells entering an exposed but not infected state, or a defectively infected state as referred to in some literature. The effect of drug treatment is seamlessly integrated into the model by connecting the biological relationship between infected cells, viral load, and infection rate, to the number of cells in the neighborhood. Some other aspects of HIV dynamics that have not been simulated in CA models before are the latently infected and exposed but not infected cell states. These novel approaches bring us one step closer to a high fidelity model for HIV dynamics and drug treatment.

The results shown here indicate that this model has great potential to provide insight into HIV infection and drug treatment dynamics. The effectiveness of the drug treatment schemes can be quantitatively assessed when tabulated and displayed as histograms of the infection stages. Control input can be easily added to the model to simulate dosage adjustment and therapy scheduling. The preliminary results show that the dosage level can be gradually regulated to stabilize the elevation of the viral load and the declination of the healthy cell count so that the infection can be controlled at a manageable level. These are positive indications that this model is very promising in evaluating and designing different drug therapy regimens.

To continue this effort, refinements and improvements should be made to incorporate more biological features of the disease into the model. Future research should include analysis of the parameter space and its effects on model performance, and clinical data should be used to verify the 
a

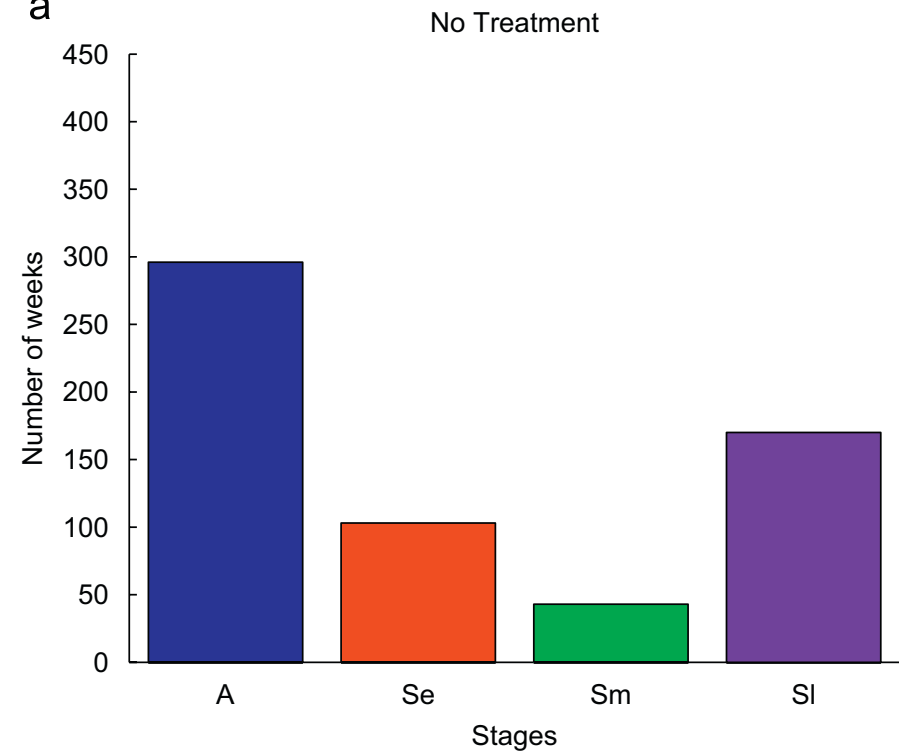

C

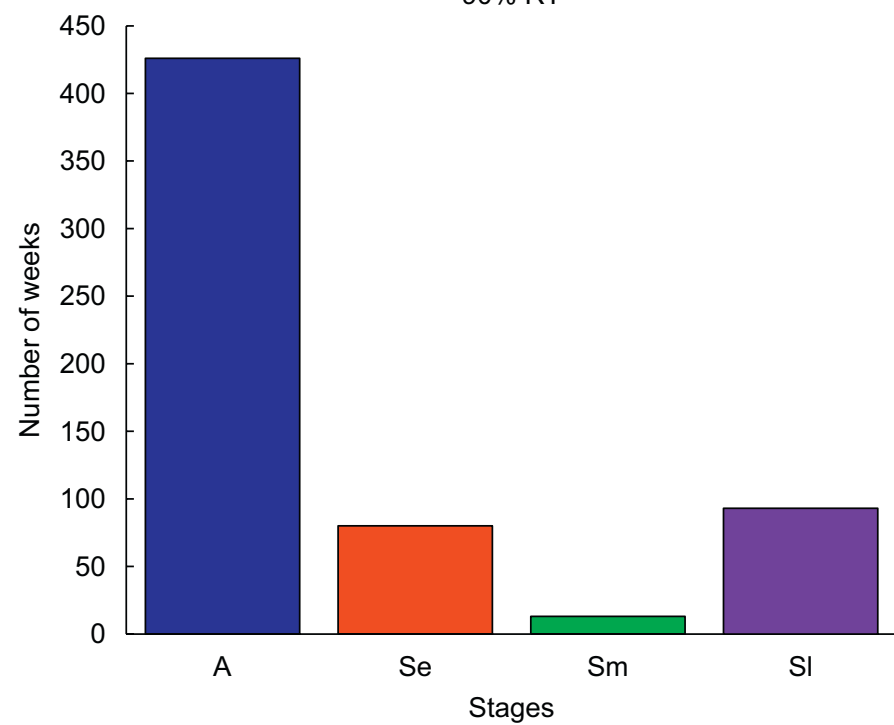

b

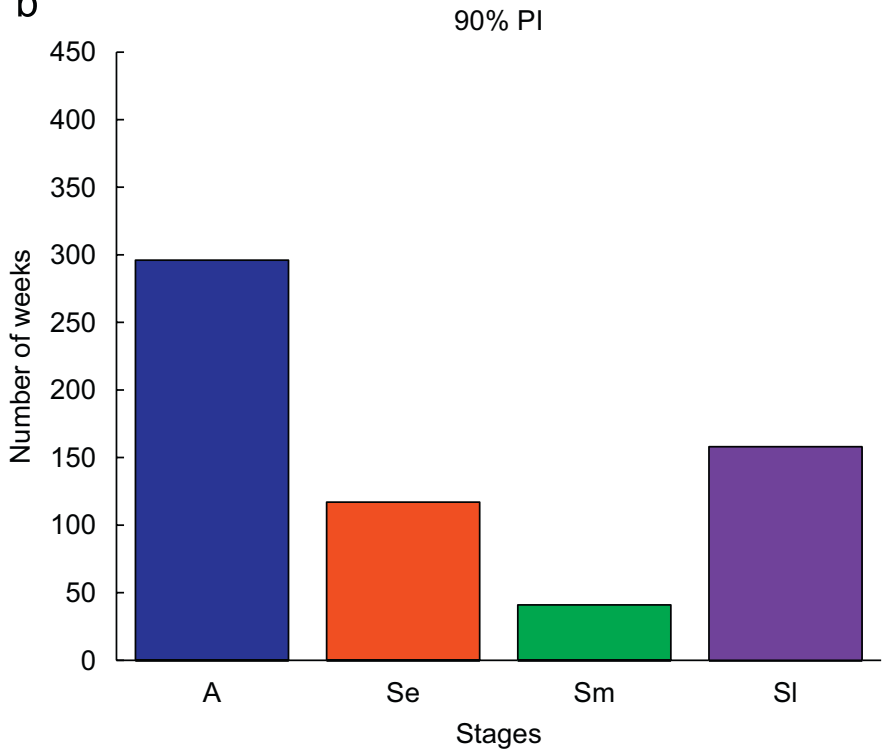

d

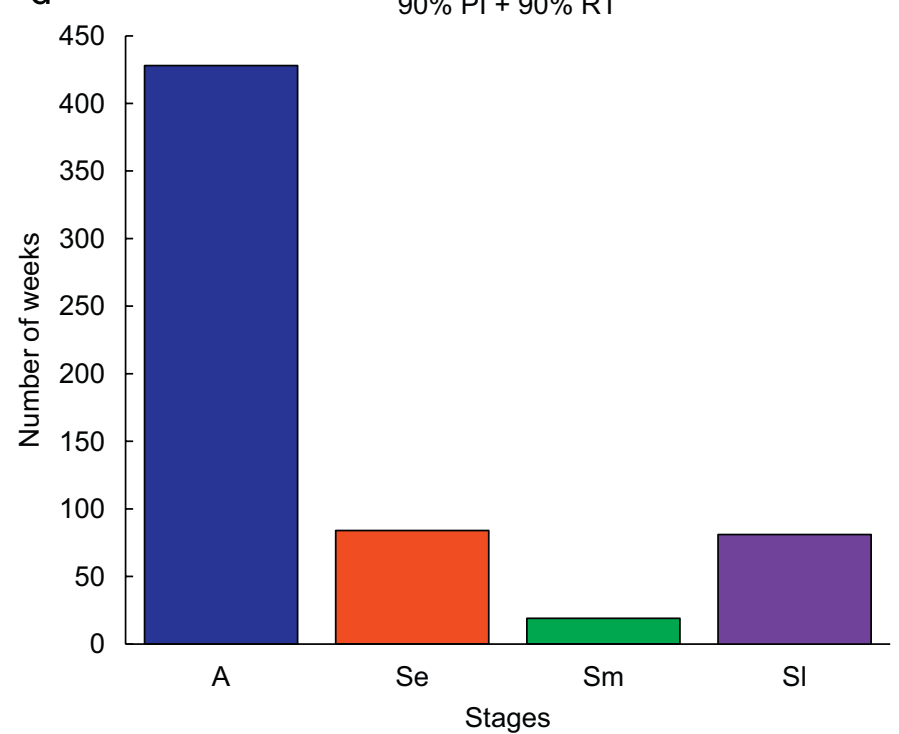

Fig. 7. Histogram of HIV infection stages for selected treatment schemes. (a) No treatment. (b) Treatment with protease inhibitor efficacy at $90 \%$. (c) Treatment with RT inhibitor efficacy at $90 \%$. (d) Combination therapy with protease inhibitor and RT inhibitor efficacy both at $90 \%$. Labels for the histograms are: A — asymptomatic stage; Se — early symptomatic stage; Sm—-medium symptomatic stage; and $\mathrm{Sl}$ - late symptomatic stage.

model results. Efforts should also be directed to make the model capable of quantitatively characterizing the disease progression and drug treatment. Future simulations will be useful to search for an optimum drug treatment regimen and investigate the effect of starting time, interruption, and/or schedule periodicity of the therapy on the prognosis of the disease using this model.

\section{Acknowledgments}

The research of Abdessamad Tridane and Yang Kuang is supported in part by DMS-0436341 and DMS/NIGMS0342388 .

\section{References}

Arnaout, R.A., Nowak, M.A., Wodarz, D., 2000. HIV-1 dynamics revisited: biphasic decay by cytotoxic T lymphocyte killing? Proc. Biol. Sci. 267 (1450), 1347-1354.

Bailey, J., Blankson, J.N., Wind-Rotolo, M., Siliciano, R.F., 2004. Mechanisms of HIV-1 escape from immune responses and antiretroviral drugs. Curr. Opinion Immunol. 16 (4), 470-476.

Bonhoeffer, S., May, R.M., Shaw, G.M., Nowak, M.A., 1997. Virus dynamics and drug therapy. Proc. Natl Acad. Sci. USA 94 (13), 6971-6976.

Culshaw, V., Ruan, S., 2000. A delay-differential equation model of HIV infection of CD4(+) T-cells. Math. Biosci. 165, 27-39.

De Boer, R.J., Perelson, A.S., 1989. Target cell limited and immune control models of HIV infection: a comparison. J. Theor. Biol. 190, 201-214. 
Dixit, N.M., Perelson, A.S., 2005. HIV dynamics with multiple infections of target cells. Proc. Natl Acad. Sci. 102 (23), 8198-8203.

Fauci, A.S., 2003. HIV and AIDS: 20 years of science. Nat. Med. 9, 839-843.

Ganguly, N., Sikdar, B.K., Deutsch, A., Canright, G., Chaudhuri, P.P., 2003. A survey on cellular automata. Technical Report, Centre for High Performance Computing, Dresden University of Technology.

Grossman, Z., Feinberg, M.B., Paul, W.E., 1998. Multiple modes of cellular activation and virus transmission in HIV infection: a role for chronically and latently infected cells sustaining viral replication. Proc. Natl Acad. Sci. 95, 6314-6319.

Hammer, S.M., Katzenstein, D.A., Hughes, M.D., Gundacker, H., Schooley, R.T., Haubrich, R.H., et al., 1996. A trial comparing nucleoside monotherapy with combination therapy in HIV-infected adults with CD4 cell counts from 200 to 500 per cubic millimeter. N. Engl. J. Med. 335, 1081-1090.

Havlir, D.V., Strain, M.C., Clerici, M., Ignacio, C., Trabattoni, D., Ferrante, P., Wong, J.K., 2003. Productive infection maintains a dynamic steady state of residual viremia in human immunodeficiency virus type 1-infected persons treated with suppressive antiretroviral therapy for five years. J. Virol. 77 (20), 11212-11219.

Ho, D.D., 1997. Dynamics of HIV-1 replication in vivo. J. Clin. Invest. 99 (11), 2565-2567.

Hood, L.E., Weissmann, I.L., Wood, W.B., Wilson, J.H., 1984. Immunology. The Benjamin/Cummings Publishing Company, Menlo Park, CA.

Kirschner, D., 1996. Using mathematics to understand HIV immune dynamics. Not. Am. Math. Soc. 43 (2), 191-202.

Kirschner, D., Webb, G.F., 1998. A mathematical model of combined drug therapy of HIV infection. J. Theor. Med. 1, 25-34.

Lafeuillade, A., Hittinger, G., Chadapaud, S., 2000. Rational basis for an immune intervention in the treatment of primary HIV-1 infection. AIDS Rev. 2, 84-92.

Muller, V., Vigueras-Gómez, J.F., Bonhoeffer, S., 2002. Decelerating decay of latently infected cells during prolonged therapy for human immunodeficiency virus type 1 infection. J. Virol. 76 (17), 8963-8965.
Nelson, P.W., Perelson, A.S., 2002. Mathematical analysis of delay differential equation model of HIV-1 infection. Math. Biosci. 179, 73-94.

Nelson, P.W., Murray, J.D., Perelson, A.S., 2000. A model of HIV-1 pathogenesis that includes an intracellular delay. Math. Biosci. 163, 201-215.

Nowak, M.A., May, R.M., 2000. Virus Dynamics: Mathematical Principles of Immunology and Virology. Oxford University Press, New York.

Nowak, M.A., Anderson, R.M., Boerlijst, M.C., Bonhoeffer, S., May, R.M., McMichael, A.J., 1996. HIV evolution and disease progression. Science 274, 1008-1010.

Pantaleo, G., Fauci, A.S., 1996. Immunopathogenesis of HIV infection. Annu. Rev. Microbiol. 50, 825-854.

Perelson, A., Kirschner, D., De Boer, R., 1993. Dynamics of HIV infection of CD4 + T cells. Math. Biosci. 114, 81-125.

Perelson, A., Neumann, A., Markowitz, M., Leonard, J., Ho, D., 1996. HIV dynamics in vivo: virion clearance rate, infected cell life span and viral generation time. Science 271, 1582-1586.

Perelson, A.S., Nelson, P.W., 1999. Mathematical analysis of HIV-1 dynamics in vivo. SIAM Rev. 41 (1), 3-44.

Ramratnam, B., Mittler, J.E., Zhang, L., Boden, D., Hurley, A., Fang, F., Macken, C.A., Perelson, A.S., Markowitz, M., Ho, D.D., 2000. The decay of the latent reservoir of replication-competent HIV-1 is inversely correlated with the extent of residual viral replication during prolonged anti-retroviral therapy. Nat. Med. 6, 82-85.

Sloot, P., Chen, F., Boucher, C., 2002. Cellular automata model of drug therapy for HIV infection. Lecture Notes in Computer Science, vol. 2493. Springer, Berlin, pp. 282-293.

Strain, M.C., Levine, H., 2002. Comment on "Dynamics of HIV infection: a cellular automata approach”. Phys. Rev. Lett. 89 (21), 198-205.

Weisstein, E.W. Moore neighborhood. From MathWorld-A Wolfram web resource. 〈http://mathworld.wolfram.com/MooreNeighborhood.html〉.

Wodarz, D., Nowak, M.A., 1998. Mathematical models of virus dynamics and resistance. J. HIV Ther. 3, 36-41.

Zorzenon dos Santos, R.M., Coutinho, S., 2001. Dynamics of HI infection: a cellular automata approach. Phys. Rev. Lett. 87 (16), $102-168$. 